\title{
Contrast-enhanced ultrasound for needle biopsy of thoracic lesions
}

\author{
JINGCHEN LIANG ${ }^{*}$, DUO WANG ${ }^{*}$, HONGXUE LI, SHENGFA ZHAO, \\ MIAO CHEN, HANG LI, ZHANLING DING, JUNJIE LIU and LIANFENG LIU \\ Department of Ultrasound, Affiliated Tumor Hospital of Guangxi Medical University, \\ Nanning, Guangxi Zhuang Autonomous Region 530021, P.R. China
}

Received September 17, 2019; Accepted June 8, 2020

DOI: $10.3892 / \mathrm{ol} .2020 .11936$

\begin{abstract}
Two-dimensional ultrasound (US) and color doppler flow imaging are associated with certain limitations in the preprocedural evaluation and design of the puncture path for biopsies of thoracic lesions, such as a poorly defined boundary between the tumor and the atelectatic lesions in central lung cancer with atelectasis. Contrast-enhanced ultrasound (CEUS) can be valuable in the preoperative evaluation of the biopsy site and in increasing the accuracy of the biopsy. The present study investigated the value of clinical application of CEUS in US-guided core needle biopsy (US-CNB) in improving the diagnostic accuracy in thoracic lesions. A total of 120 patients with first-stage thoracic lesions from the Affiliated Tumor Hospital of Guangxi Medical University who underwent US-CNB were recruited and randomnly assigned to a conventional US group $(n=66)$ and a CEUS group $(n=54)$. All patients underwent preoperative evaluation and US-guided puncture of thoracic lesions. The intergroup differences in sonographic features, biopsy duration, biopsy success rate and complications were assessed. The CEUS group had a higher rate of detection of necrotic tissue $(40.7 \%$ vs. $16.7 \%$; $\left.\chi^{2}=8.633 ; \mathrm{P}=0.003\right)$ and change of initial puncture path (48.1\%) compared with the US group. In central lung cancer with atelectasis, the ability to distinguish between tumor and atelectasis was higher in the CEUS group compared with the conventional US group (31.5 vs. $7.6 \% ; \chi^{2}=11.336$; $\mathrm{P}=0.001)$. In addition, the CEUS group had a higher puncture success $\left(96.3\right.$ vs. $\left.80.3 \% ; \chi^{2}=6.946 ; \mathrm{P}=0.008\right)$ and a
\end{abstract}

Correspondence to: Dr Lianfeng Liu or Dr Junjie Liu, Department of Ultrasound, Affiliated Tumor Hospital of Guangxi Medical University, 71 Hedi Road, Nanning, Guangxi Zhuang Autonomous Region 530021, P.R. China

E-mail: liulianfenggmu@163.com

E-mail: liujunjie197806@163.com

${ }^{*}$ Contributed equally

Key words: contrast-enhanced ultrasound, diagnosis, thoracic lesions lower complication rate $\left(3.7 \%\right.$ vs. $\left.18.2 \% ; \chi^{2}=6.041 ; \mathrm{P}=0.014\right)$ compared with the US group. CEUS can identify necrotic areas and occult tumors within atelectatic lung tissue and can be used for guiding puncture biopsy of thoracic lesions to improve the diagnostic accuracy with greater comparative clinical utility than conventional US. Pre-biopsy CEUS is especially useful for patients undergoing repeated US-CNB and those with hypovascular lesions, atelectasis or necrosis.

\section{Introduction}

Thoracic lesions include diseased tissues in the lung, mediastinum, pleura and chest wall, and are mostly lung lesions, which are primarily diagnosed as lung cancer (1). To a large extent, the diagnosis, treatment and prognosis of thoracic lesions depends on their pathological classification (2). At present, ultrasound (US), computed tomography (CT), magnetic resonance imaging, X-ray, other imaging-guided percutaneous biopsy and fiberoptic bronchoscopic biopsy are the main investigative tools for a pathological diagnosis of chest lesions (2). However, there are certain disadvantages to these investigations. A CT-guided percutaneous biopsy exposes the patient to a considerable amount of radiation without being able to dynamically display the puncture process in real time (3), resulting in possible complications. Similarly, fiberoptic bronchoscopy is associated with difficulty in accessing lumps on the pleura, chest wall and lung-adjacent peripheral pleura (4). US-guided percutaneous biopsy of thoracic lesions, especially those close to the chest wall has gradually become one of the preferential diagnostic methods for chest lesions due to its advantages of simplicity, being radiation free, providing real-time dynamic monitoring throughout the entire puncture process as well as the possibility of lesser complications $(5,6)$.

Despite the high accuracy of conventional US-guided percutaneous biopsy, there are certain limitations with regard to the preoperative evaluation and design of the puncture path in two-dimensional (2D) US and color doppler flow imaging (CDFI); this poses challenges for the identification of non-perfused areas, such as intralesional necrotic, unliquified tissues, occult tumors in atelectatic lung tissue or a poorly defined boundary between the tumor and the atelectatic lesions in central lung cancer with atelectasis $(7,8)$. These challenges impact the initial diagnosis of the lesion as well as the 
design of the biopsy path, conferring some difficulties to the procedure and resulting in poor diagnostic yield of the biopsy specimen (5).

Contrast-enhanced ultrasound (CEUS; acoustic contrast) involves the use of injectable contrast agents to enhance backscatter echo, thereby significantly intensifying the resolution, sensitivity and specificity of US diagnosis and reflecting the blood perfusion of healthy and diseased tissues (7). The sensitivity of blood flow imaging is significantly higher and unaffected by the noise of the heartbeat in CEUS compared with color doppler US $(9,10)$. In addition, CEUS can effectively identify both necrotic and viable tissues within the lesion, as well as provide valuable information for the accurate design and execution of the preoperative puncture biopsy (11). However, there is no established gold standard diagnostic method for the evaluation of thoracic lesions. Therefore, the present study was conducted to evaluate the comparative clinical application value of CEUS against that of conventional US in thoracic lesions with an aim to identify a novel standard technique for clinical US-guided percutaneous core-needle biopsy (CNB).

\section{Materials and methods}

Patients. This prospective, non-randomized controlled study included patients who were diagnosed with chest-occupying lesions between July 14, 2016 and January 31, 2018 at the Affiliated Tumor Hospital of Guangxi Medical University (Nanning, Guangxi). The inclusion criteria of the patients were as follows: i) With a lesion in the chest detected on CT examination that was clearly visualizable with US; ii) with complete clinical data; iii) who underwent an US-guided percutaneous biopsy; iv) with biopsy results or results of surgical or other pathological biopsy contributing to the final diagnosis; and v) with records of short-term complications (including hemoptysis, bleeding, pneumothorax and chest pain) after CNB and after follow up for 4-8 h postoperatively. Exclusion criteria were as follows: i) Patients with poor cooperation and unable to perform image-guided biopsies; ii) patients with contraindications for the use of CEUS, such as impaired cardiopulmonary function or known allergic reactions; and iii) patients with a bleeding tendency (prothrombin activity, $<40 \%$; international standardized ratio, $>1.7$; platelets, $<40,000 / \mathrm{ml}$ ). Following screening, a total of 120 patients with first-stage thoracic lesions were included in the present study and assigned to the US group $(n=66)$ and CEUS group $(n=54)$ based on their expressed preference. The present study was approved by the Ethics Committee of the Affiliated Tumor Hospital of Guangxi Medical University (Nanning, Guangxi; approval no. LW2018055) and was conducted in accordance with the principles of the Declaration of Helsinki and its amendments. All the patients provided written informed consent prior to being enrolled in the study.

Radiological investigations. All patients underwent a chest $\mathrm{CT}$ examination to indicate the location of the lesion prior to their initial US-CNB. For the US examination, patients were placed in the supine, lateral, or prone position with the chest surface region of the probable lesion fully exposed. Following this the thoracic region was scanned from different angles to ensure the probe was placed as close to the chest
Table I. Final diagnosis of CEUS group $(n=54)$ and US group $(n=66)$ after biopsy.

\begin{tabular}{lrc}
\hline Characteristics of lesions & CEUS group, $\mathrm{n}$ & US group, $\mathrm{n}$ \\
\hline Malignant & 35 & 42 \\
Lung squamous carcinoma & 9 & 10 \\
Lung adenocarcinoma & 15 & 16 \\
Adenosquamous carcinoma & 1 & 2 \\
Small cell carcinoma & 3 & 3 \\
Large cell carcinoma & 1 & 0 \\
Lymphoma & 2 & 5 \\
Malignant mesothelioma & 1 & 0 \\
Neuroendocrine carcinoma & 0 & 1 \\
Metastatic carcinoma & 3 & 5 \\
Benign & 19 & 24 \\
Inflammatory pseudotumor & 6 & 7 \\
Pneumonia with consolidation & 6 & 8 \\
Solitary fibrous tumor & 0 & 2 \\
Schwannoma & 1 & 0 \\
Tuberculosis & 6 & 7 \\
Total & 54 & 66 \\
\hline
\end{tabular}

CEUS, contrast-enhanced ultrasound; US, ultrasound.

wall as possible. Gaseous and rib obstructions were avoided and the size, shape and internal structure of the lesion was carefully observed and recorded. The presence of liquefaction necrosis and the positional relationship with the surrounding lung tissue were also observed and recorded using US. The vascular supply of the lesion was observed using CDFI. All US images were recorded in the color Doppler Aplio500/Aplio400 (Toshiba Corp.).

In the CEUS group, a color Doppler Aplio500/Aplio400 (Toshiba Corp.) with a frequency of $3.5 \mathrm{MHz}$ and a mechanical index of 0.12-0.18 was used with sulfur hexafluoride microbubbles (SonoVue ${ }^{\circledR}$; Bracco) as the contrast agent. For each set of images, a 2.4-ml bolus (injected in 3-5 sec) of contrast agent suspension was injected (prepared by dissolving $24.98 \mathrm{mg}$ dry powder in $5 \mathrm{ml}$ normal saline) through a peripheral venous catheter inserted into an antecubital vein followed by a $5 \mathrm{ml}$ saline flush. A stopwatch was started with the contrast injection and dynamic images were recorded. These lesions were examined continuously for at least $180 \mathrm{sec}$ and included part of the normal peripheral parenchyma in the same ultrasound scan in order to examine the enhancement of the lesion and the surrounding normal lung in real time. If normal lung parenchyma could not be included in the same imaging scan, or the lesion was located at the basis of the lung, the surrounding chest wall or the liver (lesion in right lung) or spleen (lesion in left lung) was examined contemporaneously to the lesion (12). In the case of unsatisfactory vascular imaging, the contrast injection was repeated (inter-injection interval $>10 \mathrm{~min}$ ). Following US, 2 experienced sonographers from the Affiliated Tumor Hospital of Guangxi Medical University played back the video of the CEUS and recorded the enhancement level, 
Table II. Comparison of imaging features and biopsy success rate in the CEUS group (n=54) and US group ( $\mathrm{n}=66)$.

\begin{tabular}{|c|c|c|c|c|}
\hline Characteristics & CEUS group & US group & $\mathrm{Z}\left(\chi^{2}\right)$-value & P-value \\
\hline Size, $\mathrm{cm}$ & $3.0(3.0-4.0)$ & $3.4(3.0-5.0)$ & -1.437 & 0.151 \\
\hline Necrosis, \% (n/total) & $40.7(22 / 54)$ & $16.7(11 / 66)$ & 8.633 & 0.003 \\
\hline Atelectasis, \% (n/total) & $31.5(17 / 54)$ & $7.6(5 / 66)$ & 11.336 & 0.001 \\
\hline Punctures times, $\mathrm{n}$ & $3(3-4)$ & $3(3-5)$ & -1.574 & 0.116 \\
\hline Success rate, $\%$ (n/total) & $96.3(52 / 54)$ & $80.3(53 / 66)$ & 6.946 & 0.008 \\
\hline Complications, \% (n/total) & $3.7(2 / 54)$ & $18.2(12 / 66)$ & 6.041 & 0.014 \\
\hline
\end{tabular}

CEUS, contrast-enhanced ultrasound; US, ultrasound. Data are presented as median (quartile spacing) as the Shapiro-Wilk test showed that the size of the lesion and the number of punctures were all skewed $(\mathrm{P}>0.05)$.

enhancement pattern and vascular shape of the lesions. Differences, if any, in the evaluation of the US images were resolved by mutual discussion to arrive at a consensus.

Fine needle biopsy. Preoperatively, a routine examination of blood and coagulation function was performed. For the biopsy, patients were positioned appropriately on the basis of the location of the lesion. In the US group, the areas with rich blood supply or hypoechogenicity were selected as the biopsy site (echo-free areas were avoided). In the CEUS group, the enhanced areas were selected (unenhanced areas were avoided) and following determination of the location of the lesion, the most appropriate needle path and puncture depth were planned.

The CNB procedure was undertaken as follows: Routine disinfection with Maokang complex iodine skin disinfectant, draping, local anesthesia with $2 \%$ lidocaine, use of a specific probe for the puncture, reconfirmation of the puncture site, path, and depth and insertion of the biopsy needle $\left(\right.$ Bard $^{\circledR}$ Magnum $^{\circledR}$; C.R. Bard; BD Biosciences) through the skin into the deep layers of the chest wall. The patient was asked to hold his/her breath, the needle was quickly inserted into the lesion under US guidance and the biopsy gun was triggered immediately. The puncture needle was pulled out quickly after cutting the tissue and then the biopsy was completed. In general, biopsies were obtained using 2-4 core needles, although an additional 1-2 needles were used if necessary. In case of unsatisfactory CNBs with inadequate tissue samples, additional punctures with repositioning of needle direction were performed. The tissue sampling was considered successful if the biopsy specimen met the requirements for histopathological diagnosis. The tissue specimens were fixed using $10 \%$ formalin at room temperature and were sent to the pathology department for a routine histological examination 30 min following fixing. Following the removal of the needle, the puncture point was covered with a sterile gauze bandage and pressure was applied. Patients were advised bed rest for 4-8 h after the biopsy, their vital signs were monitored, and complications were noted (such as bleeding, hemoptysis, chest tightness, chest pain, pneumothorax and air embolisms).

Final diagnosis. A comprehensive diagnosis of all biopsy specimens was done by 2 experienced pathologists from The Affiliated Tumor Hospital of Guangxi Medical University (Nanning, China), and if the diagnosis of the biopsy specimen was true in the subsequent analysis, the diagnosis was considered to be true. Malignant tumors were identified using surgical specimens or subsequent re-biopsies obtained from other means, such as biopsy under fiberoptic bronchoscope. The benign diagnosis was considered to be true positive if the imaging examination confirmed that the lesion disappeared or shrank following treatment during the 6 month follow-up period. A false negative diagnosis was given using the following criteria: i) The diagnosis of the biopsy specimen was considered to be negative for a malignant tumor and subsequently confirmed by surgery or re-biopsy as a malignant tumor; ii) the biopsy specimen was insufficient; ii) or there was no clear descriptive diagnosis, such as chronic inflammation, necrosis, muscle tissue, etc. The diagnostic accuracy of the biopsy was defined as the percentage of lesions with true positive results in the initial biopsy (13).

Statistical analysis. Data were subjected to statistical analysis using SPSS version 19.0 J (IBM Corp.). The Shapiro-Wilk method was used to determine if data was normally distributed or not. Unpaired data with skewed distribution were analyzed using the Mann-Whitney $U$ test and the results were expressed as the median (quartile). The Wilcoxon signed-rank test was used for comparison of paired continuous data with skewed distribution. Data were expressed as a rate (\%) on analysis with the Pearson's $\chi^{2}$ test, used for categorical data. $\mathrm{P}<0.05$ was considered to indicate a statistically significant difference.

\section{Results}

Demographic information of patients. A total of 120 patients were included in the final analysis of the present study. Of these, 66 cases were assigned to the US group (males, 46; females, 20; mean age \pm SD, 52.9 \pm 15.6 ; and age range, $17-74$ years) and 54 cases to the CEUS group (male, 40; female, 14; mean age \pm SD, 54.6 \pm 13.9 ; age range, 15-81 years). Subjects were assigned to each study arm on the basis of their individual preference to undergo either conventional US or CEUS.

Histopathological diagnosis of CEUS and US groups. Details of the histopathological diagnosis of both groups are presented in Table I. In the CEUS group $(n=54), 35$ malignant lesions 
A

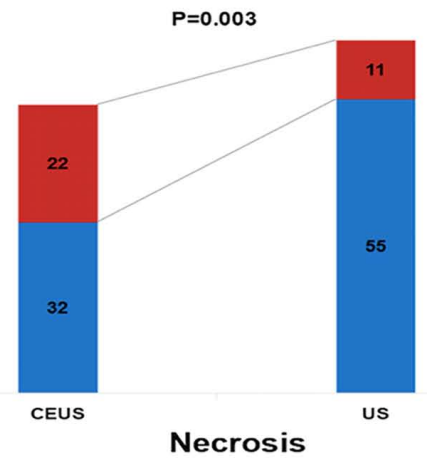

C

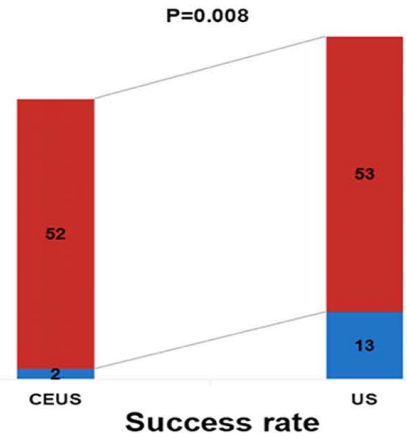

B

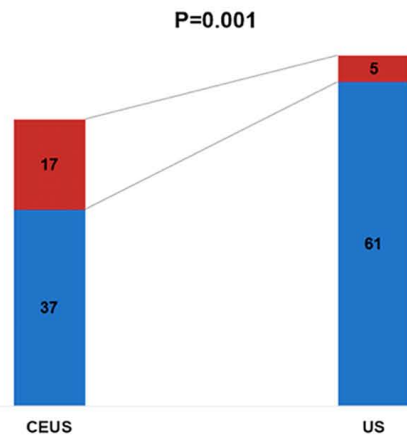

Atelectasis

D

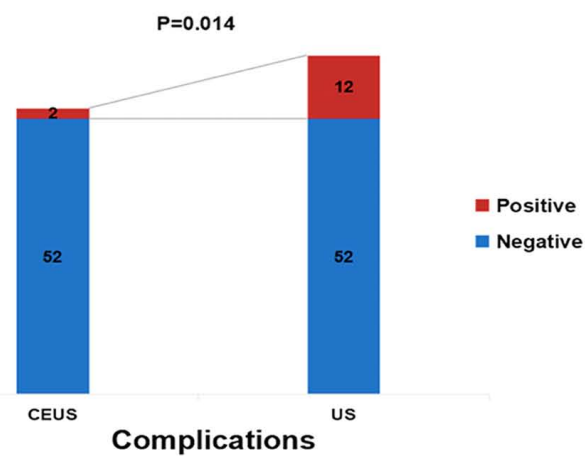

Figure 1. Comparison of characteristics between the CEUS and US groups. (A) Necrosis, (B) atelectasis, (C) success rate and (D) complications. CEUS, contrast-enhanced ultrasound; US, ultrasound.
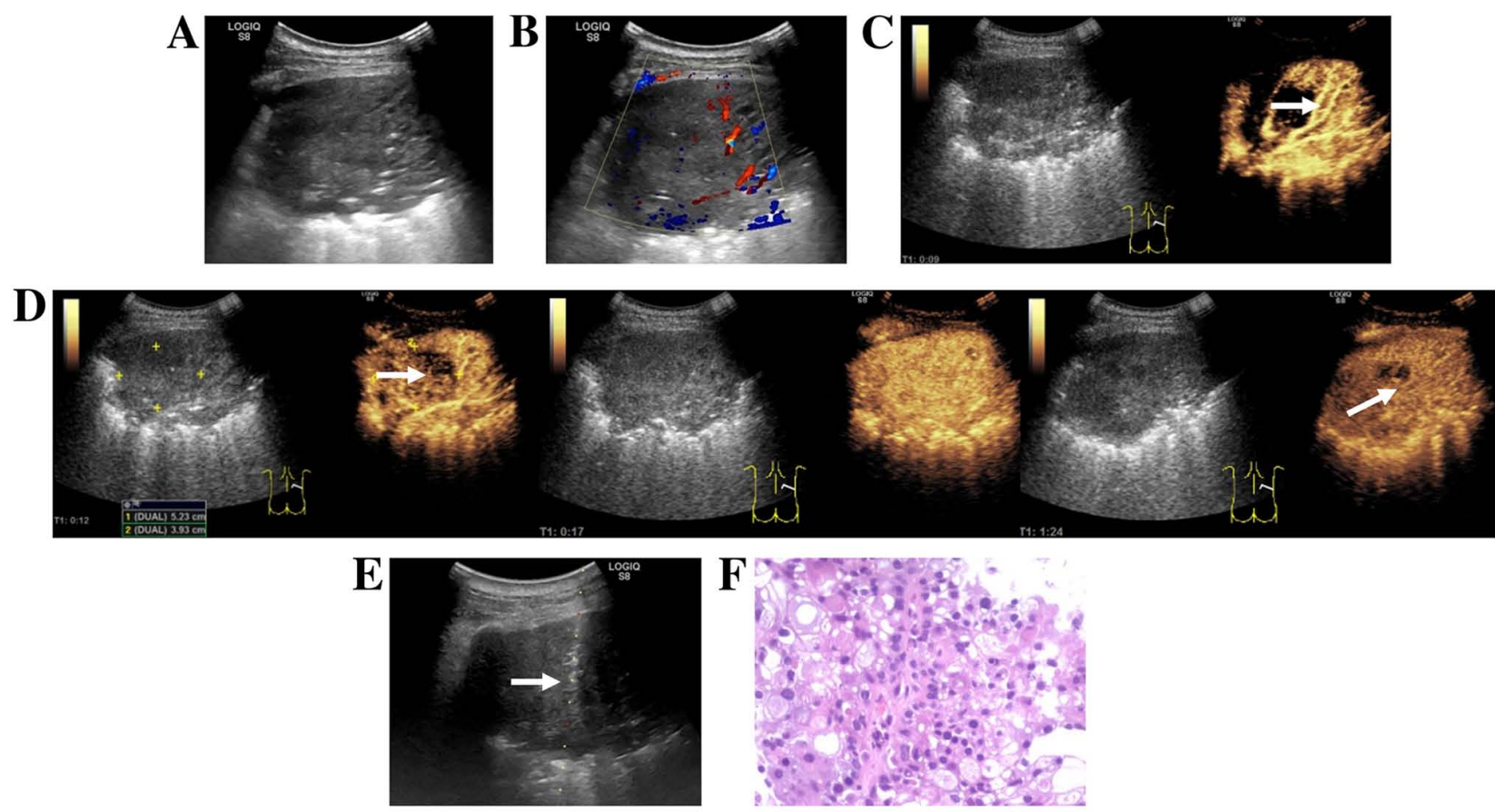

Figure 2. Images from a 52-year-old man with a history of cough and dyspnea. (A) Routine ultrasound revealed large hypoechoic areas in the inferior lobe of the right lung. (B) Color Doppler flow imaging revealed strip-color flow signals in the right side of the lesion. (C) CEUS obtained 9 sec after injection of SonoVue ${ }^{\circledR}$ (Braggo) revealed a rapid and uniform enhancement of the right region of the lesion, as well as a dendritic vascular structure, suggesting atelectasis (white arrow). (D) CEUS revealed the enhancement pattern of the tumor (white arrow) was 'slow in and fast out' compared with that of the atelectasis in the right lung. The demarcation between the tumor and atelectasis was clear. (E) CEUS-guided core-needle biopsy of the tumor area (white arrow). (F) Hematoxylin and eosin staining of histopathological biopsy specimen revealed poorly differentiated carcinoma (original magnification, x200). The H\&E staining results were obtained using routine histopathology. CEUS, contrast-enhanced ultrasound.

were identified (lung squamous cell carcinoma, 9; lung adenocarcinoma, 15; adenosquamous carcinoma, 1 ; small cell lung carcinoma, 3; lung large cell carcinoma, 1; lymphoma, 2; malignant mesothelioma, 1; and metastatic carcinoma, 3) 

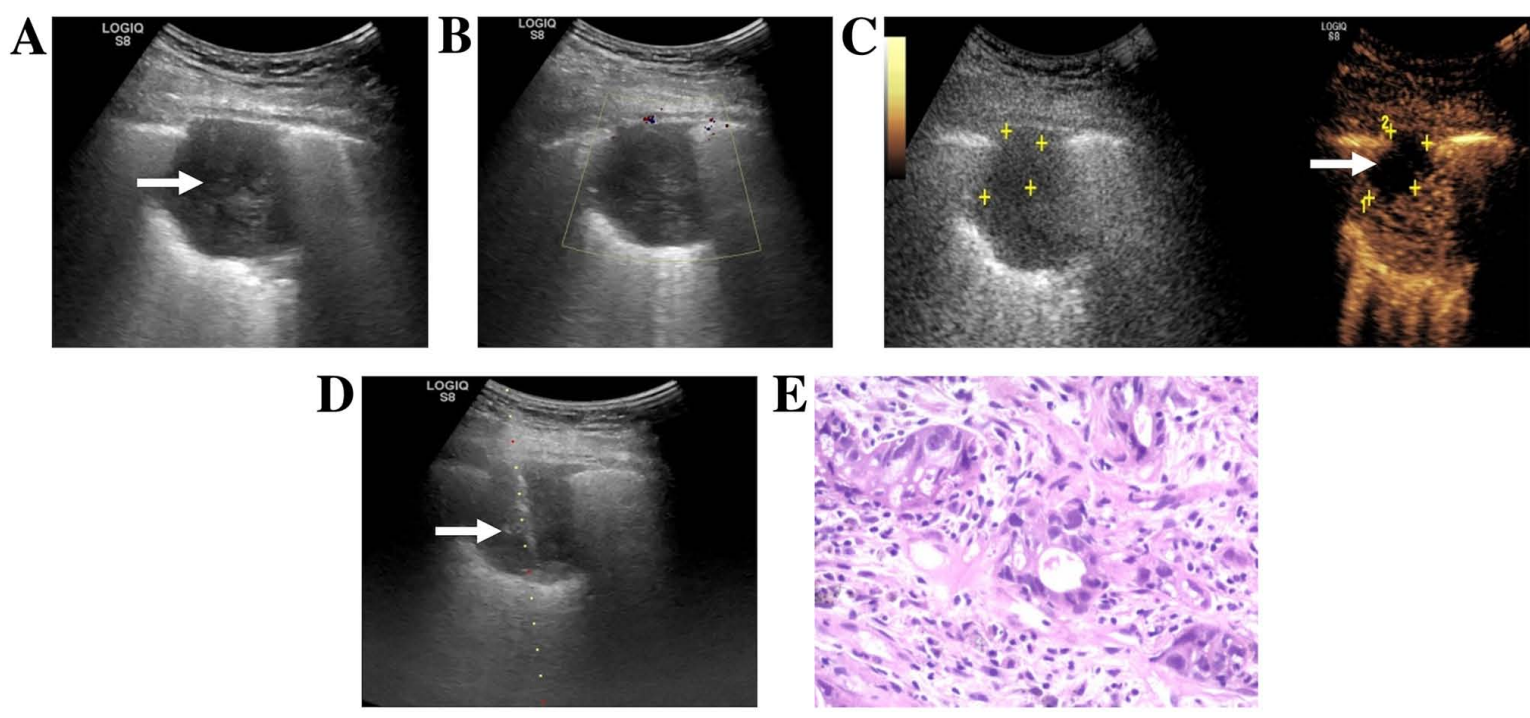

Figure 3. Images from a 47-year-old man with a history of chest pain and hemoptysis. (A) Routine ultrasound showed a hypoechoic lesion (white arrow) in the superior lobe of the right lung. (B) Color Doppler flow imaging showed no color flow signal around or inside the nodule. (C) CEUS obtained 42 sec after an injection of SonoVue ${ }^{\circledR}$ (Braggo) showed irregular necrosis in the anterior part of the lesion (white arrow and yellow markings). (D) US-guided transthoracic biopsy passed through the necrotic area and targeted the enhanced area (white arrow). (E) Hematoxylin-eosin staining of histopathological biopsy specimen revealed an adenosquamous carcinoma (original magnification, x200). The H\&E staining results were obtained using routine histopathology. CEUS, contrast-enhanced ultrasound; US, ultrasound.

Table III. Initial puncture path was changed during US-guided transthoracic biopsy after CEUS examination in CEUS group.

Characteristics

\begin{tabular}{ll}
\hline Necrosis & $31.5(17 / 54)$ \\
Atelectasis & $16.6(9 / 54)$ \\
Total & $48.1(26 / 54)$ \\
\hline
\end{tabular}

and 19 benign lesions (inflammatory pseudotumor, 6; pneumonia, 6; schwannoma, 1; and tuberculosis, 6) (Table I).

In the US group, 42 malignant lesions were detected (lung squamous cell carcinoma, 10; lung adenocarcinoma, 16; adenosquamous carcinoma, 2; small cell carcinoma, 3; lymphoma, 5; neuroendocrine carcinoma, 1; and metastatic carcinoma, 5) and 24 benign lesions (inflammatory pseudotumor, 7; pneumonia, 8; solitary fibrous tumor, 2; and tuberculosis, 7) (Table I).

Comparison of imaging features in the CEUS and US groups. Shapiro-Wilk test demonstrated that the size of the lesion and the number of punctures were all skewed $(\mathrm{P}>0.05)$. There were no significant differences among groups in lesion size and puncture times $(\mathrm{P}>0.05$; Table II). The CEUS group had a higher rate of detection of necrotic tissue compared with the US group (40.7 vs. 16.7\%; $\chi^{2}=8.633 ; \mathrm{P}=0.003$; Table II; Fig. 1A). In patients with central lung cancer and atelectasis, the CEUS group exhibited greater ability to discriminate between the tumor and atelectasis, compared with the conventional US group (31.5 vs. $7.6 \% ; \chi^{2}=11.336 ; \mathrm{P}=0.001$; Table II; Fig. $\left.1 \mathrm{~B}\right)$. In addition, in the CEUS group, the demarcation between the tumor and
Table IV. Post hoc analysis of the failure of the puncture in the US and CEUS groups.

\begin{tabular}{lcc}
\hline Cause & CEUS group & US group \\
\hline Necrotic tissue & 0 & 7 \\
Lung tissue and skeletal & 0 & 3 \\
muscle tissue & & \\
Insufficient organization & 1 & 0 \\
Complications & 0 & 2 \\
Poor patient cooperation & 1 & 1 \\
Total & 2 & 13 \\
\hline
\end{tabular}

CEUS, contrast-enhanced ultrasound; US, ultrasound.

atelectasis, as well as the area for puncture biopsy were more clearly defined compared with the US group (Fig. 2). The H\&E staining images in Figs. 2 and 3 were obtained by routine histopathology.

Comparison of biopsy success rate and complication rate in the CEUS and US groups. In 48.1\% (26/54) of the patients in the CEUS group, the initial puncture path was changed during US-guided transthoracic biopsy due to the presence of necrotic tissue or atelectasis tissue in the lesion by CEUS examination, including 17 cases $(31.5 \%)$ due to necrotic tissue (Fig. 3) and 9 cases (16.6\%) due to pulmonary atelectasis (Table III). The CEUS group had a higher CNB success rate (96.3 vs. $80.3 \% ; \chi^{2}=6.946 ; P=0.008$; Table II; Fig. $1 C$ ) and a lower complication rate compared with the US group ( 3.7 vs. $18.2 \% ; \chi^{2}=6.041 ; P=0.014$; Table II; Fig. 1D). In the CEUS group, there was 1 case of hemorrhage and 1 case of chest pain whereas the US group there were 5 cases of 


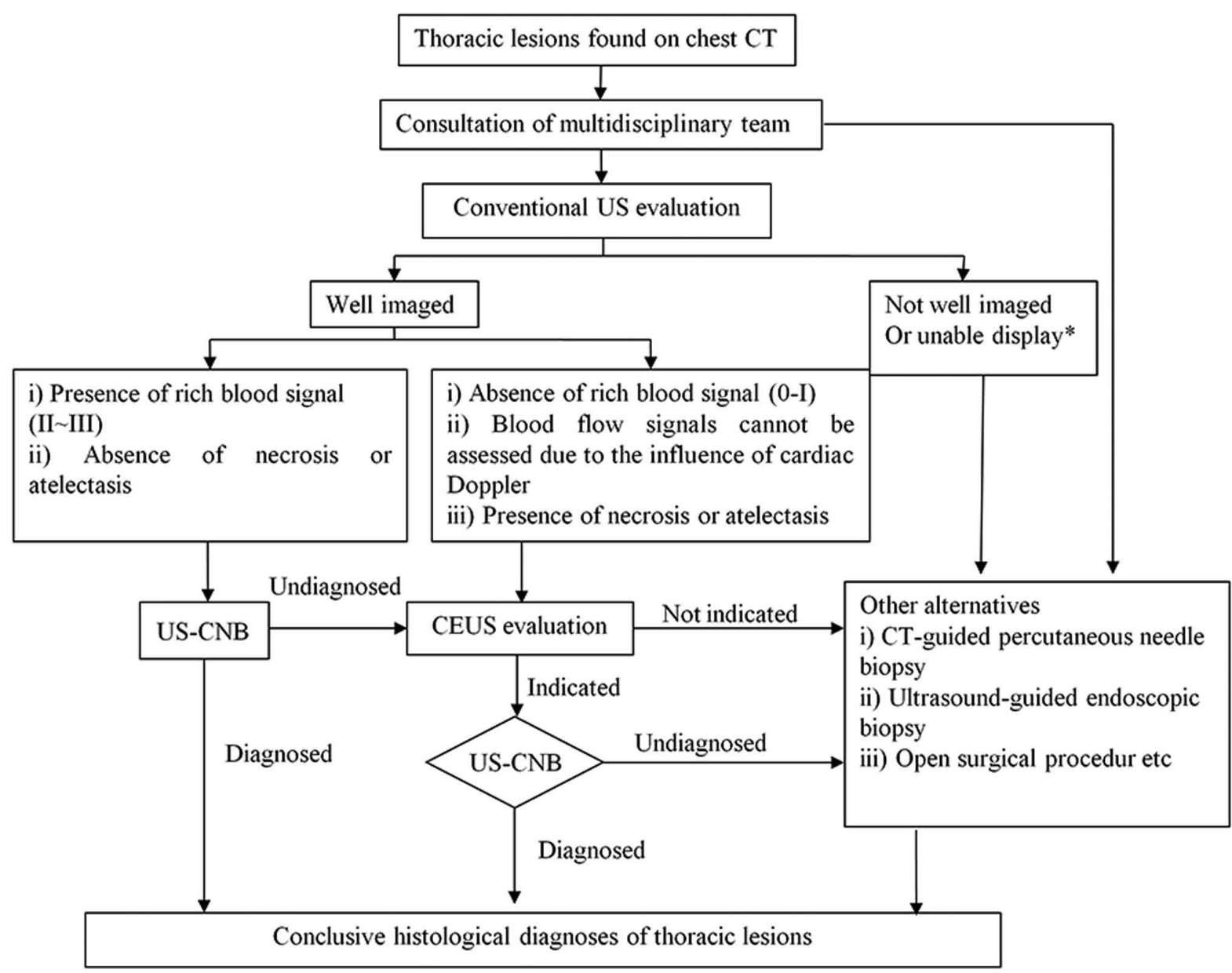

Figure 4. Proposal for integration of CEUS into the algorithm for evaluation of thoracic lesions. US-CNB, ultrasound-guided core needle biopsy; CEUS, contrast-enhanced ultrasound; US, ultrasound.'Unable display' means conventional ultrasound cannot show the lesions due to the influence of gas in the lung tissue in front of the lesion.

hemoptysis, 2 cases of chest pain, 4 cases of pneumothorax and 1 case of hemorrhage (Table II). All the complications observed in the present study were reported in previous studies of conventional ultrasound-guided biopsy $(3,8,14)$ and resolved with conservative management including hemostasis, oxygen inhalation and bed rest. There were no reports of serious complications, such as severe hemopneumothorax requiring closed thoracic drainage.

Post hoc analysis of puncture failure in the CEUS and US groups. A post hoc analysis was performed to evaluate the cause of puncture failure in the 2 patients in the CEUS group, and insufficient tissue specimen and poor patient cooperation were identified as the causes (Table III). A similar analysis of 13 patients with puncture failure in the US group revealed that 7 cases had necrotic tissue in biopsy specimens, 3 had healthy lung and muscle tissues, 2 developed intraprocedural complications during puncture and 1 had poor patient cooperation (Table IV).

\section{Discussion}

In recent years, US-guided percutaneous biopsy has emerged as one of the main investigative techniques to obtain histopathological specimens from chest lesions (7). Increasingly, it is becoming the first-choice investigation in chest lesions because of its advantages of procedural simplicity, safety and effectiveness, without the risk of exposure to ionizing radiation $(5,6)$ Despite its high success rate and fewer complications, conventional US has certain limitations in the preoperative evaluation of chest lesions and the design of the puncture path (7). For example, although conventional 2D US can recognize liquefaction within lesions, it may only poorly identify necrotic and un-liquified tissues (7). In addition, although CDFI can broadly observe lesion vascularity, it has low sensitivity and the results of the observation are easily influenced by numerous factors, including the angle between the sound wave and the blood vessel, false negative readings by slow blood flow and noise interference due to the heartbeat (14). Conventional US cannot easily distinguish between occult tumors and atelectatic lung tissue $(8,14)$. These aforementioned factors may decrease the accuracy of the biopsy results $(7,15)$. A rebiopsy can improve the diagnostic accuracy, but with an increase in costs, time to treatment, risk of complications and patient anxiety, amongst other factors.

CEUS is a vascular imaging method that can effectively display real-time blood perfusion in healthy and diseased tissues and provide more diagnostic information compared with conventional US for a pre-biopsy assessment (4). Sartori et al (11) reported the first case of peripheral lung tumor with large necrotic tissue successfully guided by CEUS 
in 2004. Previous studies have demonstrated that CEUS can positively identify necrotic areas in lung lesions, despite a lack of consensus on the ability of CEUS to differentiate between benign and malignant thoracic lesions $(4,16)$. The results of the present study demonstrated that the CEUS group had a higher rate of detection of necrotic tissue (40.7 vs. $16.7 \%$ ) compared with the US group. In central lung cancer with atelectasis, the ability to distinguish between tumor and atelectasis was higher in the CEUS group compared with that in the conventional US group (31.5 vs. 7.6\%). In addition, in $48.1 \%$ of the patients in the CEUS group, the initial puncture path was changed during US-guided transthoracic biopsy due to the presence of necrotic tissue or atelectasis tissue in the tumor by CEUS examination; the CEUS group had a higher puncture success (96.3 vs. $80.3 \%$ ) and a lower complication rate (3.7 vs. $18.2 \%$ ) compared with those in the US group. The present results are similar to those reported in previous studies $(7,8,11)$, suggesting that CEUS may identify the necrotic areas in the lesions, as well as the tumors hidden in the atelectasis. The inference of a rationale for this is based on a literature review (17). Microvessels are present in viable tissues of the lesion, but absent in necrotic areas. Therefore, regardless of whether CEUS or super micro-imaging is undertaken in the contrast mode, the contrast agent will enter viable tissue, but not the necrotic tissue (4). Therefore, contrast imaging can effectively distinguish between viable tissue and necrotic areas within lesions (11). The vascular supply to malignant tumors of the chest primarily originates from the bronchial artery, whereas the peritumoral lung tissue receives dual vasculature from both the pulmonary and bronchial arteries (4). The injected contrast agent initially enters the pulmonary artery prior to entering the bronchial artery; therefore, the viable lung tissue enhances before the diseased tissue and this is an important basis to distinguish lung atelectasis from tumor tissue (7). In addition, the findings of the present study demonstrated earlier initial enhancement of lung tissue compared with diseased tissue which corresponds to the histopathological basis of the lesion. The heterogeneity of tumoral tissue with the associated unevenness of vascularity results in heterogeneous enhancement, which is an important basis for distinguishing between tumor tissue and atelectatic lung tissue (16). In addition, atelectatic pulmonary tissue has a regular and branched vasculature, whereas tumor vasculature is distorted and irregular (8). Therefore, CEUS prior to a CNB can effectively increase the success rate of puncture and minimize the probability of a repuncture. However, to avoid overdiagnosis and the associated treatment, CEUS should not be routinely recommended to all patients. A pre-puncture CEUS is recommended for patients with chest lesions, with hypovascular lesions (0-I blood flow, on Adler grading (18) or indeterminable blood supply (due to cardiac Doppler effects, for example) in the presence of atelectasis or necrosis who are scheduled to undergo a repeat US-CNB. This recommendation is explained in Fig. 4.

The present study has several limitations. Firstly, the small sample size is insufficient to generate adequate statistical power to validate the current findings. Secondly, the study groups were not randomized. Thirdly, potential confounders, such as the body mass index of patients and poor cooperation were not accounted for, which likely have an impact on the results of the study. The findings of the present study need to be validated in well-designed, randomized, large cohort, multi-center prospective studies.

In conclusion, the present study demonstrated that CEUS can identify necrotic areas and occult tumors within atelectatic lung tissue and can be used for guiding puncture biopsy of thoracic lesions to improve diagnostic accuracy with greater clinical utility compared with conventional US. Pre-biopsy CEUS is particularly useful for patients undergoing repeated US-CNB and those with hypovascular lesions, atelectasis or necrosis.

\section{Acknowledgements}

Not applicable.

\section{Funding}

The present study was supported by grants from the Youth Science Foundation of Guangxi Medical University (grant no. GXMUYSF201725), Competence Improvement Project of Young and Middle-aged Teachers in Colleges and Universities in Guangxi (grant no. 2018K Y0118) and National Natural Science Foundation of China (grant no. 81701721).

\section{Availability of data and materials}

All data generated or analyzed during this study are included in this published article.

\section{Authors' contributions}

LL and JJL designed the study and revised the manuscript. DW and ZD participated in designing and conducting the research. JCL conceived the research and contributed to the writing of the manuscript. HXL and SZ collected and analyzed the data. MC and HL analyzed the data and interpreted the results. All authors have read and approved the manuscript.

\section{Ethics approval and consent to participate}

The present study was approved by the Ethics Committee of the Affiliated Tumor Hospital of Guangxi Medical University (Nanning, Guangxi; approval no. LW2018055). All the patients provided written informed consent prior to being enrolled in the study.

\section{Patient consent for publication}

Not applicable.

\section{Competing interests}

The authors declare that they have no competing interests.

\section{References}

1. Pilleron S, Sarfati D, Janssen-Heijnen M, Vignat J, Ferlay J, Bray F and Soerjomataram I: Global cancer incidence in older adults, 2012 and 2035: A population-based study. Int J Cancer 144: 49-58, 2019. 
2. Anna ML, David B and Lauren T: Diagnosing lung cancer: The complexities of obtaining a tissue diagnosis in the era of minimally invasive and personalised medicine. J Clin Med 7: 163, 2018.

3. Yamamoto N, Watanabe T, Yamada K, Nakai T, Suzumura T, Sakagami K, Yoshimoto N, Sato K, Tanaka H, Mitsuoka S, et al: Efficacy and safety of ultrasound (US) guided percutaneous needle biopsy for peripheral lung or pleural lesion: Comparison with computed tomography (CT) guided needle biopsy. J Thorac Dis 11: 936-943, 2019.

4. Piscaglia F, Nolsøe C, Dietrich CF, Cosgrove DO, Gilja OH, Nielsen MB, Albrecht $\mathrm{T}$, Barozzi L, Bertolotto $M$, Catalano O, et al: The EFSUMB guidelines and recommendations on the clinical practice of contrast enhanced ultrasound (CEUS) Update 2011 on non-hepatic applications. Ultraschall Med 33: 33-59, 2012.

5. Choi YR, An JY, Kim MK, Han HS, Lee KH, Kim SW, Lee KM and Choe KH: The diagnostic efficacy and safety of endobronchial ultrasound-guided transbronchial needle aspiration as an initial diagnostic tool. Korean J Intern Med 28: 660-667, 2013.

6. Jarmakani M, Duguay S, Rust K, Conner K and Wagner JM Ultrasound versus computed tomographic guidance for percutaneous biopsy of chest lesions. J Ultrasound Med 35: 1865-1872, 2016.

7. Song W, Wei Y, Hui Z, Qian X and Yan K: The role of contrast-enhanced ultrasound in selection indication and improveing diagnosis for transthoracic biopsy in peripheral pulmonary and mediastinal lesions. Biomed Res Int 2015: 231782, 2015.

8. Lei Z, Lou J, Bao L and Lv Z: Contrast-enhanced ultrasound for needle biopsy of central lung cancer with atelectasis. J Med Ultrason 45: 461-467, 2018.

9. Ramnefjell M, Aamelfot C, Aziz S, Helgeland L and Akslen LA: Microvascular proliferation is associated with aggressive tumour features and reduced survival in lung adenocarcinoma. J Pathol Clin Res 3: 249-257, 2017.
10. Lu R, Meng Y, Zhang Y, Zhao W, Wang X, Jin M and Guo R Superb microvascular imaging (SMI) compared with conventional ultrasound for evaluating thyroid nodules. BMC Med Imaging 17: 65, 2017.

11. Sartori S, Nielsen I, Trevisani L, Tombesi P, Ceccotti P and Abbasciano V: Contrast-enhanced sonography as guidance for transthoracic biopsy of a peripheral lung lesion with large necrotic areas. J Ultrasound Med 23: 133-136, 2004.

12. Yi D, Feng M, Wang WP, Ji ZB and Fan PL: Value of contrast-enhanced ultrasound in guidance of percutaneous biopsy in peripheral pulmonary lesions. Biomed Res Int 2015: 531507, 2015.

13. Yi D, Feng M, Wen PW, Zheng BJ and Fan PL: Contrast-enhanced US-guided percutaneous biopsy of anterior mediastinal lesions. Diagn Interv Radiol 23: 43-48, 2017.

14. Guo YQ, Liao XH, Li ZX, Chen YY, Wang SD, Wang JH, Liao X and Luo Y: Ultrasound-guided percutaneous needle biopsy for peripheral pulmonary lesions: Diagnostic accuracy and influencing factors. Ultrasound Med Biol 44: 1003-1011, 2018.

15. Jeon KN, Bae K, Park MJ, Choi HC, Shin HS, Shin S, Kim HC and Ha CY: US-guided transthoracic biopsy of peripheral lung lesions: Pleural contact length influences diagnostic yield. Acta Radiol 55: 295-301, 2014.

16. Görg C, Bert T and Görg K: Contrast-enhanced sonography for differential diagnosis of pleurisy and focal pleural lesions of unknown cause. Chest 128: 3894-3899, 2005.

17. Görg C, Bert T and Kring R: Contrast-enhanced sonography of the lung for differential diagnosis of atelectasis. J Ultrasound Med 25: 35-39, 2006.

18. Adler DD, Carson PL, Rubin JM and Quinn-Reid D: Doppler ultrasound color flow imaging in the study of breast cancer: Preliminary findings. Ultrasound Med Biol 16: 553-559, 1990.

This work is licensed under a Creative Commons

Attribution-NonCommercial-NoDerivatives 4.0

International (CC BY-NC-ND 4.0) License. 